\title{
Comprehensive Learning Particle Swarm Optimization (CLPSO) for Multi-objective Optimal Power Flow
}

\author{
Meysam Rahmati ${ }^{1}$, Reza Effatnejad ${ }^{2 *}$ and Amin Safari ${ }^{3}$ \\ 'Department of Electrical Engineering, Islamic Azad University, Ahar branch, Ahar, Iran; Mysam.pse@gmail.com \\ 2Department of Electrical Engineering, Islamic Azad University-Karaj branch, Karaj, Iran; reza.efatnejad@kiau.ac.ir \\ ${ }^{3}$ Department of Electrical Engineering, Faculty of Engineering, Azarbaijan Shahid Madani University, \\ Tabriz, Iran; safari@azaruniv.edu
}

\begin{abstract}
The Optimal Power Flow (OPF) problem has been widely used in power system operation and planning for determining electricity prices and amount of emission. This paper presents a Comprehensive Learning Particle Swarm Optimization (CLPSO) algorithm to solve the highly constrained multi-objective OPF involving conflicting objectives, considering fuel cost and emission level functions. The proposed technique has been carried out on IEEE 30-bus test system. The results demonstrate the capability of the proposed CLPSO approach to generate well-distributed Pareto optimal non-dominated solutions of multi-objective OPF problem. The results show that the approaches developed are feasible and efficient.
\end{abstract}

Keywords: Comprehensive Learning, Multi-objective Optimization, Particle Swarm Optimization, Optimal Power Flow

\section{Introduction}

One of the major problems in the operation of power system is optimal power flow. The aim of this problem is minimizing the fuel cost per unit of production to produce a certain amount of power equal to the demand. The optimal power flow must be done in such a way that the other constraints on production rates and associated constraints will also satisfy. Optimal power flow determines the optimal combination of real power generation, voltage magnitudes, compensator capacitors and transformer tap position to minimize the specific objective function like total generation cost in power systems. In other word, optimal power flow is combination of economic dispatch and power flow that. In fact power flow is a constraint for OPF problem. By renovation of the structure and developing the power system, because of existence of local optimum points from general solutions, the OPF has become more complicated too because finding general solution for OPF problem has more difficult too. The OPF is powerful optimization software to find combination of power control variable, a desired objective function is minimized. The mentioned conditions make the OPF problem a large scale non-linear constrained optimization problem. It appears that the term "optimal power flow" was first introduced by Dommel and Tinney in $1968^{1}$. A literature survey reveals that various numerical optimization techniques have been used for the OPF problem, such as Non-Linear Programming (NLP) ${ }^{2}$, Linear Programming $(L P)^{3}$, Newton methods ${ }^{4}$, quadratic programming 5 , integer programming ${ }^{6}$ and many heuristic methods have been used to solve a OPF problem such as simulated annealing $^{7}$, Genetic Algorithm (GA) ${ }^{8}$, Differential Evolution algorithm (DE) ${ }^{9}$, Particle Swarm Optimization (PSO) ${ }^{10}$.

However, according to the public sensitivities and legal limitations for environmental pollutions, power distribution between thermal power plants, do not surveyed only with economic feature and the power should 
distributed with a manner between distribution units that produced pollution has its minimum level. In this case, the given objective function of pollution is considered in OPF problem. Thus we deal with two objectives, that those two objectives has contradiction with each other and it may, by reducing one of them another one will increased and vice versa. Then we should reach to a compromise solution between two objectives. Emission of toxic gases such as carbon dioxide, nitrogen dioxide and sulfur dioxide at power plants, the researchers thought that the optimal power flow based not only on cost but also reduced pollution to be resolved. Pollution diffusion not only has human damages, but also cause to harming to the materials, agriculture and becoming the earth planet warmer. In this paper, two of the most important emissions of nitrogen dioxide and sulfur dioxide are studied. Recently, pay attention to the environmental protection is increased dramatically ${ }^{11}$, in this regard some studies have focused on environmental protection such as predict and control the pollutant emission of potential industrial installations ${ }^{12}$.

Multi-objective optimization is a design methodology that optimizes a group of objective functions systematically and simultaneously. There are several techniques that have been considered in articles to solve the multiobjective problem. Recently, these methods have received much interest due to the development of multi-objective evolutionary search strategies. Multi-objective OPF solved with modified Shuffle Frog Leaping Algorithm (SFLA) ${ }^{13}$.

This paper proposes the application of PSO and CLPSO to solve the multi-objective OPF problem. Standard test systems of IEEE 30-bus system have been employed to carry out the simulation study. Both the PSO and CLPSO methods perform well in such systems for OPF and give satisfactory results. The simulation results demonstrate that the proposed method is capable of obtaining accurate and acceptable solutions. Also, to validate the obtained results, they are compared with original SLFA, GA and PSO methods. Simulation results demonstrate that CLPSO algorithm is superior in compression the other algorithms.

\section{Problem Formulation}

The goal of multi-objective OPF is to minimize two conflicting objectives, which are the fuel cost and pollutants emission, while satisfying operating and loading constraints. Generally the problem is formulated as:

\subsection{Problem Objectives}

\subsubsection{Minimization of Fuel Cost}

The total fuel cost $F\left(P_{g}\right)$ can be expressed as:

$F\left(P_{g}\right)=\sum_{i=1}^{n_{g}} a_{i}+b_{i} P_{g i}+c_{i} P_{g i}^{2}$

where, $p_{g}$ is the generation active power of power unit and $a_{i}, b_{i}$ and $c_{i}$ are fuel cost coefficients of $i^{\text {th }}$ unit.

\subsubsection{Minimization of Emission}

The atmospheric pollutants caused by fossil-fueled thermal units can be modeled separately. The emission function can be presented as the sum of all types of emission considered, such as $\mathrm{NO}_{x}, \mathrm{SO}_{x}$ thermal emission, etc., with suitable pricing or weighting on each pollutant emitted. In the present study, two important types of emission gases are taken into account. The generated $\mathrm{NO}_{x}$ and $\mathrm{SO}_{x}$ level is defined as follow ${ }^{14}$ :

$E\left(P_{g}\right)=\sum_{i=1}^{N_{G}}\left(\gamma_{i} P_{G i}^{2}+\beta_{i} P_{G i}+\alpha_{i}+\xi_{i} \exp \left(\lambda_{i} P_{G i}\right)\right)$

$($ ton $/ h)$

where, $E\left(P_{g}\right)$ is the total emission cost $($ ton $/ h)$ and $\gamma_{i} \beta_{i}, \xi_{i}$ and $\lambda_{i}$ are the emission coefficients of $i^{\text {th }}$ unit, while $\gamma_{i}\left(\right.$ ton $\left./ h M W^{2}\right), \beta_{i}($ ton $/ h M W), \alpha_{i}($ ton $/ h)$ are related to $S O_{x}$ and $\xi_{i}($ ton $/ h), \lambda_{i}(1 / M W)$ are related to $N O_{x}$ respectively ${ }^{15}$.

\subsection{Objective Constraints}

\subsubsection{Power Balance Constraints}

The total power generation must cover the total power demand and the power loss. This implies solving the power flow problem, which has equality constraints on active and reactive power at each bus as follows:

$P_{i}=P_{g i}-P_{d i}=\sum_{j=1}^{n} V_{i} V_{j}\left(G_{i j} \cos \theta_{i j}+\beta_{i j} \sin \theta_{i j}\right)$
$Q_{i}=Q_{g i}-Q_{d i}=\sum_{j=1}^{n} V_{i} V_{j}\left(G_{i j} \sin \theta_{i j}-\beta_{i j} \cos \theta_{i j}\right)$

where, $i=1,2, \ldots, n$ and $\theta_{i j}=\theta_{i}-\theta_{j}$.

\subsubsection{Generation Capacity Constraints}

For stable operation, real and reactive power output of each generator is restricted by lower and upper limits as follows:

$P_{g i \min } \leq P_{g i} \leq P_{g i \max }$

$Q_{g \text { imin }} \leq Q_{g i} \leq Q_{g i \max }$ 


\subsubsection{Security Constraints}

For secure operation, transmission line active loading $P_{i j}$ is restricted by its upper limit. Load buses voltage magnitude is also limited between lower and upper limits:

$$
\begin{aligned}
& \left|P_{i j}\right| \leq P_{i j \max } \\
& V_{i \min } \leq V_{i} \leq V_{i \max } \\
& i=1,2, \ldots, n l
\end{aligned}
$$

where, $n l$ is the number of load buses and $P_{i j}$ is the power that flows between bus-i and bus-j. $V_{i \max }$ and $V_{\text {imin }}$ voltages are the maximum and minimum valid voltages for each bus, respectively. $P_{i j \max }$ is the maximum power that flows through the branch. $P_{g i \max }$ and $P_{g i \min }$ are the maximum and minimum active power values of $\mathrm{i}^{\text {th }}$ bus, respectively. $Q_{g \text { imax }}$ and $Q_{g i \min }$ are maximum and minimum reactive power values of $i^{\text {th }}$ bus.

\section{Fuzzy-based Mechanism for Best Compromise Solution}

We can obtain not only a unique optimal solution but also we can achieve a set of Pareto optimal solutions by benefiting from CLPSO method in optimization of the above formulated objective functions which will result in a situation that for the purpose of improving one objective, the other objectives must be sacrificed. Of course for benefiting from this methods practically a solution must be selected that would assist us to realize our aims and purposes which can be name as the best compromise solution. Inaccuracy of the decision maker's judgment is considered as the most challenging element of the trade off decision. For the purpose of obtaining a candidate Pareto optimal solution for decision theory fuzzy set theory was implemented ${ }^{16-18}$. Using Pareto optimal set a fuzzy-based procedure will be presented to come to this conclusion that a Pareto optimal solution would be the best compromise solution. Often using the experiences and intellectual awareness of the decision maker a membership function will be introduced for each of the objective functions. In this research for every objective function a simple linear membership function was determined. The membership function is defined as follows ${ }^{18}$ :

$$
\mu=\left\{\begin{array}{lc}
1, & F_{i} \leq F_{i}^{\min } \\
\frac{F_{i}^{\max }-F_{i}}{F_{i}^{\max }-F_{i}^{\min },} & F_{i}^{\min }<F_{i}<F_{i}^{\max } \\
0, & F_{i} \geq F_{i}^{\max }
\end{array}\right.
$$

where, $F_{i}^{\min }$ and $F_{i}^{\max }$ are the maximum and minimum values of the $i^{\text {th }}$ objective function, respectively. Therefore, the membership function represents the degree of achievement of the original objective function as a value between 0 and 1 with $\mu_{i}=1$ as completely satisfactory and $\mu_{i}=0$ as unsatisfactory. Such a linear membership function represents the decision maker's fuzzy goal of achievement, and at the same time scales the original objective functions with different physical units into the measure of $0-1$. Figure 1 illustrates a typical shape of the membership function. For each non-dominated solution $k$, the normalized membership function $\mu^{k}$ is calculated as:

$$
\mu^{k}=\frac{\sum_{i=1}^{n} \mu_{i}^{k}}{\sum_{j=1}^{m} \sum_{k=1}^{n} \mu_{i}^{j}}
$$

where, $m$ is the number of non-dominated solutions and $n$ is the number of objective functions. The best compromise solution is the one having the maximum of $\mu^{k}$. As a matter of fact, arranging all solutions in Pareto optimal set in descending order according to their membership function will provide the decision maker with a priority list of non-dominated solutions. This will guide the decision maker in view of the current operating conditions.

\section{Description of CLPSO Algorithm}

Lately, cooperative PSO has become increasingly popular among the researchers and this method separates the solution vector into small vectors, where each sub-vector is optimized by a separate PSO. Again one more parameter called 'split factor' will control the performance of this algorithm. Premature convergence is still the major deficiency of the most PSO based algorithms despite the fact

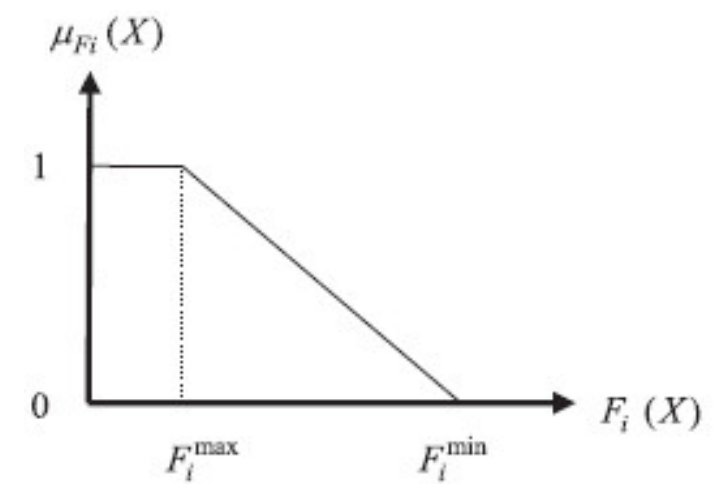

Figure 1. Membership functions for objective function. 
that there are numerous PSO variants. In the original PSO, pbest and gbest will assist each particle to learn from them simultaneously and the social learning factor is limited to only gbest. In addition to the above mentioned matters, even if the current gbest is far from the global optimum all particles in the swarm learn from the gbest. In the PSO, the personal best experience and group best experience are indicated pbest and gbest, respectively. Every particle in the swarm is updated using (12) and (13)

$$
\begin{aligned}
V_{j d}^{(k+1)}= & w V_{j d}^{(k)}+c_{1} \times \text { rand } \times\left(\text { Pbest }_{j d}-X_{j d}^{(k)}\right) \\
& +c_{2} \times \text { rand } \times\left(\text { gbest }_{d}-X_{j d}^{(k)}\right) \\
X_{j d}^{(k+1)}= & X_{j d}^{(k)}+V_{j d}^{(k+1)}, j=1,2, \ldots, m
\end{aligned}
$$

In such conditions, in case the search environment is complicated with numerous local solutions, it is possible for the particles to be easily absorbed and trapped into an inferior local optimum.

It is possible for a particle that has discovered the value relating to the global optimum in one dimension that it would have a low fitness value as a result of the poor solutions in other dimensions because of the fact that the fitness value of a particle is decided by all dimensions. In this situation it is possible to lose this good genotype. A new learning strategy is presented for the purpose of preventing this from happening. There are two major differences in comparison with several current PSO variants ${ }^{19}$ :

(1) Generally every dimension of a particle in general learns from a different pbest for different dimensions for a few iterations instead of learning from the pbest and gbest for all dimensions.

(2) Every dimension of a particle learns from just one exemplar for a few iterations instead of learning from two exemplars, namely, the pbest and gbest in all iterations in the original PSO found in Eq. (12),. In CLPSO method, for each particle, in addition its own pbest, other particles' pbests are used as exemplars. Potentially every particle learns from all particles' pbests in the swarm. No one is aware of the fact that which dimensions of particular particle's pbest are good and which are bad meanwhile the search procedure. Thus, there is equal chance for every dimension of a particle to be learnt by other particles. Some dimensions of other particles' pbests are randomly selected on the basis of a probability Pc, named learning probability, as social exemplars to be learnt from, for each particle, while other dimensions learn from its pbest, as cognitive exemplar. In the following, a different velocity updating is used for each particle as follows ${ }^{20}$ :

$$
V_{j d}^{(k+1)}=w \times V_{j d}^{(k)}+\operatorname{rand} \times\left(\text { Pbest }_{(f i) d}-X_{j d}\right)
$$

Where, the decision depends on Pc and Pbest ${ }_{(f i) d}$ may be any particle's pbest or its own pbest. This is the job of Pc to determine that which dimension will follow the particle's own pbest and which dimension will learn from the other particle's pbest. Every particle has its own Pc, which may be different from that of other particles. a random number is produced for every dimension of particle $j$. This dimension will learn from its own pbest in case the random number is larger than Pc of ${ }^{\text {th }}$ particle, otherwise it will learn from another particle's pbest. When a dimension of one particle learns from other particle's pbest is as follows: (1) two particles are randomly selected out of the population, which does not include the particle being updated, (2) the fitness values of these two particles' pbest are compared, and (3) then the winner's pbest is used as the exemplar for that dimension. One dimension is randomly chosen to learn compulsorily from other particle's pbest in case all exemplars of a particular particle are its own pbest. An annealing procedure will determine the weighting function $\mathrm{w}$, which makes uniform search in the initial stages and very local search in the latter stages. The weighting function $w_{k}$ for kth iteration is determined by

$$
w_{k}=\frac{\left(w_{0}-0.2\right) \times\left(\max _{-} \text {iter }-k\right)}{\max _{-} \text {iter }}+0.2
$$

The velocity of each particle is clamped according to the following Eq. (16) meanwhile the procedure, In (16), $\mathrm{V}_{\max }$ is set as $0.25\left(X_{\max }-X_{\min }\right)$, after experimentation.

$$
V_{j d}^{(k)}=\min \left(V_{d}^{\max }, \max \left(-V_{d}^{\max }, V_{j d}\right)\right)
$$

\section{CLPSO Implementation in Multi-objective OPF Problem}

This section demonstrates the application of the proposed algorithm in order to solve the proposed multi-objective problem. To apply the proposed algorithm to the presented problem, the following steps should be done.

STEP 1: Define the input data.

In this step, the input data including the generator real powers, generator bus voltages, fuel cost coefficient of 
generators, emission coefficient of generators, transformer tap, reactive power of compensator capacitors, $F_{i}^{\max }$ and $F_{i}^{\max }$ are defined, it is worthwhile to note that $F_{i}^{\min }$ and $F_{i}^{\min }$ are the minimum and maximum value of each objective function, respectively, when they are optimized as single objective optimization problem.

STEP 2: Generate the initial population.

Population $=\left[\begin{array}{c}x_{1} \\ x_{2} \\ \ldots \\ x_{\text {swarm }}\end{array}\right]$
$X_{i}=\left[\begin{array}{llll}x_{i, 1}, & x_{i, 2}, & \ldots, & x_{i, N}\end{array}\right]$
$X_{i}=\left[\begin{array}{llll}V_{g i}, & P_{g i}, & Q_{c i}, & \text { tap }\end{array}\right]$

where,

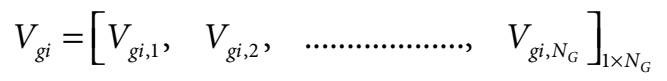

$P_{g i}=\left[\begin{array}{llllll}P_{g i, 1}, & P_{g i, 2}, \quad \ldots \ldots \ldots . . . & P_{g i, j}, \quad \ldots \ldots \ldots . . . . & P_{g i,\left(N_{G}-1\right)}\end{array}\right]_{1 \times N_{G}}$

$Q_{C i}=\left[Q_{C i, 1}, \quad Q_{C i, 2}, \quad \ldots \ldots \ldots, Q_{C i, N_{c}}\right]_{1 \times N_{c}}$

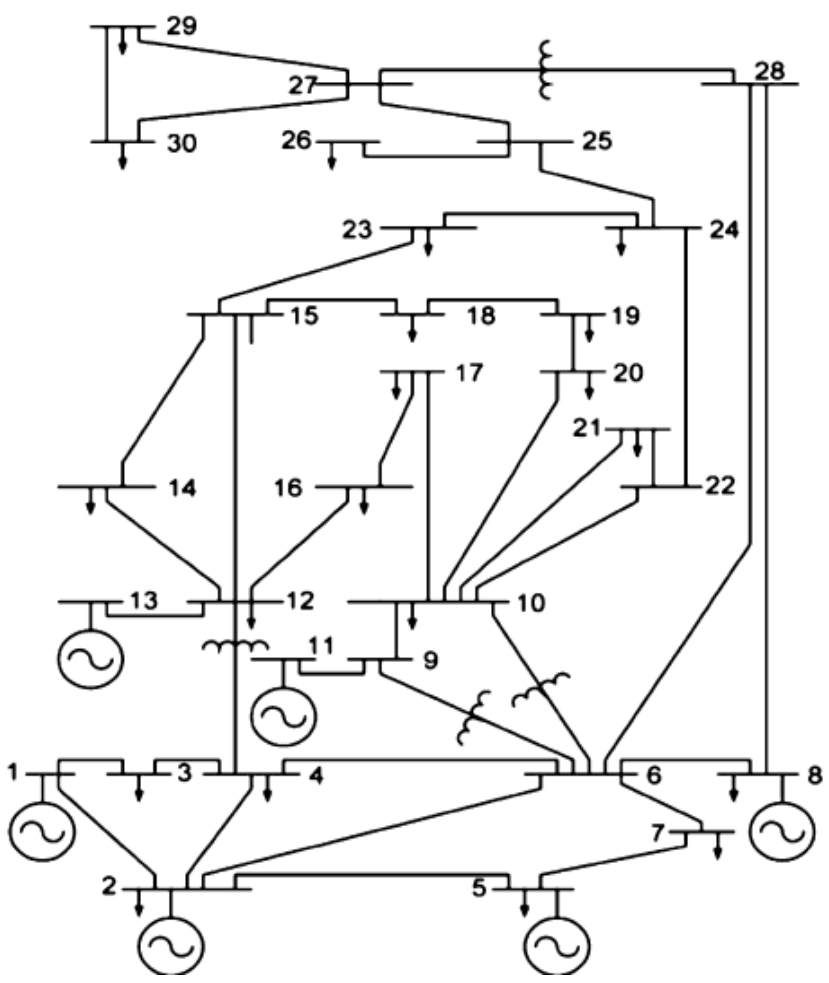

Figure 2. One line diagram of 30-bus IEEE test system. $x_{i, j}=\operatorname{rand}() *.\left(x_{j, \text { max }}-x_{j, \text { min }}\right)+x_{j, \text { min }}$

$j=1,2,3, \ldots, N_{\text {param }} ; \quad i=1,2,3, \ldots, N_{F}$

where, $x_{j}$ is the position of the jth state variable, rand(.) is a random function generator between 0 and $1, N_{C}$ is the number of compensator capacitors and $N_{F}$ is the number of the particles.

STEP 3: Convert the constraint proposed problem to an unconstraint one ${ }^{13}$.

$$
\begin{aligned}
& J(x)=\left[\begin{array}{l}
J_{1}(X) \\
J_{2}(X)
\end{array}\right]_{2 \times 1} \\
& =\left[\begin{array}{l}
F_{1}(X)+L_{1}\left(\sum_{j=1}^{N_{\text {eq }}}\left(h_{j}(X)\right)^{2}\right)+L_{2}\left(\sum_{j=1}^{N_{\text {ueq }}}\left(\left[0,-g_{j}(X)\right]\right)^{2}\right) \\
F_{2}(X)+L_{1}\left(\sum_{j=1}^{N_{\text {eq }}}\left(h_{j}(X)\right)^{2}\right)+L_{2}\left(\sum_{j=1}^{N_{\text {ueq }}}\left(\left[0,-g_{j}(X)\right]\right)^{2}\right)
\end{array}\right]
\end{aligned}
$$

The proposed multi-objective problem needs to be converted into an unconstrained problem, as follows:

where, $F_{1}(x)$ and $F_{2}(x)$ are the objective functions described in Equations (1) and (2), respectively. $N_{e q}$ and $N_{\text {ueq }}$ are the numbers of equality and inequality constraints, respectively. $h_{j}(x)$ and $g_{j}(x)$ are the equality and inequality constraints, respectively. $L_{1}$ and $L_{2}$ are the penalty factors. Since the constraints have to be met, the values of the parameters should be high. In this paper the values have been considered 10000 .

STEP 4: Compute and normalize the objective function. In this step $\mu_{i}(x)$ computed according to Equation (9).

STEP 5: Apply the Pareto method.

In this step the Pareto method is applied and all nondominated solutions are saved in the repository, also the weight factor for all non-dominate solutions is calculated based on Equation (10). The above generalized objective function is used as fitness function. Each individual's fitness value is compared with its pbest. The best value among the pbests in the swarm is denoted as gbest.

STEP 6: Velocity modification.

The velocity of each particle is modified according to (13).

STEP 7: Position modification.

The position of each individual is modified according to (12).

STEP 8: Update of individual best and global best.

If the fitness value of each individual is better than the previous pbest, the current value is set to be pbest. If the pbest is better than gbest, the value is set to be gbest. 
STEP 9: Stopping criteria.

If the number of iterations reaches set value, then the individual that generates the latest gbest is the optimal settings of control variables for the multi-objective OPF problem.

\section{Simulation Results}

The CLPSO algorithm was coded in the MATLAB environment, and executed on Intel core i3 $2.53 \mathrm{GHz}$ PC, with 4 GB of RAM. The test was performed on the IEEE 30-bus system which has 41 transmission lines and the system demand is $283.4 \mathrm{MW}$ in all simulations. The information of the system, e.g. fuel cost and emission function coefficients are given in Table 1. The one line diagram of IEEE 30-bus test system ${ }^{15}$ is shown in Figure 2.

MATPOWER $^{21}$ is a package of MATLAB m-files for solving power flow and optimal power flow problems. It is intended as a simulation tool for researchers and educators which will be easy to use and modify. In this paper we have changed MATPOWER by adding CLPSO codes in order to implement the multi-objective OPF problem in power systems. The parameters required for implementation of the CLPSO algorithm are the population size, number of iteration, $w_{0}, p_{c}$ and C. The value of these parameters is shown in Table 2. To demonstrate the effectiveness of the proposed approach, three cases with different complexity have been considered as follows:

CASE 1: Minimize total operating cost.

CASE 2: Minimize total emission.

Case 3: Minimize operating cost and emission at the same time.

Simulation results including generation fuel cost, emission level and power losses are reported in Table 3, 4 and 5.

Initially, each of the objective functions individual are examined then used The Pareto-optimal method to obtain the optimal point by optimizing the two objectives simultaneously. The results are shown in Tables 3, 4 and 5.

CASE 1: Minimize total operating cost

The minimum cost of fuel is only objective in this case. Considered results of this case are shown in Table 3 and 4. In Table 3 the results of propose algorithm are compared with $\mathrm{NLP}^{22}, \mathrm{EP}^{23}$, $\mathrm{TS}^{24}$, IEP ${ }^{25}, \mathrm{DE}-\mathrm{OPE}^{26}$ algorithms. According to Table 1, it is clear that the results of the proposed algorithm are better than those algorithms. To demonstrate the capability and power of proposed algorithms, this algorithm has been compared with new heuristic methods that results of this comparison are shown in Table 4. Algorithm has converged in 802.2240 $(\$ / \mathrm{h})$ which is the lowest cost in Table 3 and 4 . Convergence curve for this case is shown in Figure 3. Gain economically from lower fuel costs for electric power generation is very important because it saves more energy, Therefore, the algorithm is able to achieve the lowest fuel cost takes into consideration.

CAse 2: Minimize total emission

In this case, the total amount of emission will be achieved by the proposed algorithm and the results are shown in Table 4. In this table the results obtained from the proposed algorithm is compared with GA, SLF and PSO algorithm. Results of Table 5 indicate how a reduction in $\mathrm{NO}_{\mathrm{x}}, \mathrm{SO}_{\mathrm{x}}$ emission could be achieved by a change in generation dispatch schedules. In this case, the amount of pollution that can be emitted by the proposed algorithm is lower than other algorithms and it shows the ability of the proposed algorithm. Figure 4 shows the curve of convergence obtained in this case.

CASE 3: Minimize operating cost and emission at the same time

In this paper, three cases were studied to optimize. First, fuel costs and emissions for each individual were examined and in this case the objectives are optimized simultaneously. In other word, a multi-objective optimal power flow problem is solved. Pareto-optimal method is used in this case to solve compromise between the two objectives. The user based on the importance of each goal and can find different solutions. After CLPSO apply the Pareto set, the best compromise solution is selected according to Equation (8). In this regard Equation (8) is computed for all non-dominate solutions, after sorting them according to their $\mu^{k}$ value, the best solution among them considers as the best compromised solution. The best solution among the best compromise solution is studied.

For better comparison between single-objective and two-objective optimization should be noted that in the first case, when the fuel cost is the minimum amount of emissions has its maximum value because these two objectives are conflicting with each other. The result of this settlement is the compromise 891.1654 for the fuel cost and 0.2195 for emissions. Figure 5 shows the curve of the solution is compromise.

All results obtained do not violate the generation capacity constraints. It is important to note that the security constraints are satisfied for voltage magnitudes and line flows. No load bus is under its lower limit of $0.90 \mathrm{pu}$. 
Table 1. Fuel and emission coefficients of generators for IEEE 30-bus network

\begin{tabular}{llllllr}
\hline & \multicolumn{1}{c}{$G_{1}$} & $G_{2}$ & $G_{3}$ & $G_{4}$ & $G_{5}$ & $G_{6}$ \\
\hline Fuel cost coefficient & & & & & \\
$a$ & 0.00375 & 0.0175 & 0.0625 & 0.0083 & 0.025 & 0.025 \\
$b$ & 2 & 1.75 & 1 & 3.25 & 3 & 150 \\
$c$ & 0 & 0 & 0 & 0 & 0 & 0 \\
Emission coefficient & & & & & 0.05151 \\
$\gamma$ & 0.06490 & 0.05638 & 0.04586 & 0.03380 & 0.04586 & -0.05555 \\
$\beta$ & -0.05554 & -0.06047 & -0.05094 & -0.03550 & -0.05094 & 0.06131 \\
$\alpha$ & 0.04091 & 0.02543 & 0.04258 & 0.05326 & 0.04258 & 0.00001 \\
$\xi$ & 0.0002 & 0.0005 & 0.000001 & 0.002 & 0.000001 & 6.667 \\
$\lambda$ & 2.857 & 3.333 & 8.000 & 2.000 & 8.000 & \\
\hline
\end{tabular}

Table 2. Summary of parameters selected

\begin{tabular}{lrlll}
\hline Population size & $w_{0}$ & $\mathrm{c}$ & $\boldsymbol{p}_{c}$ & NO. of iteration \\
\hline 100 & 0.9 & 1 & 4 & $\begin{array}{l}100 \text { for emission function } \\
200 \text { for cost function }\end{array}$ \\
\hline
\end{tabular}

Table 3. Comparison of fuel cost obtained by proposed algorithm and other algorithms in lectures

\begin{tabular}{|c|c|c|c|c|c|c|}
\hline & NLP[22] & $\mathrm{EP}[23]$ & $\mathrm{TS}[24]$ & IEP [25] & DE-OPF[26] & CLPSO \\
\hline$P G_{1}(M W)$ & 176.26 & 173.848 & 176.04 & 176.2358 & 176.009 & 174.747 \\
\hline$P G_{2}(M W)$ & 48.84 & 49.998 & 48.76 & 49.0093 & 48.801 & 49.496 \\
\hline$P G_{5}(M W)$ & 21.51 & 21.386 & 21.56 & 21.5023 & 21.334 & 21.109 \\
\hline$P G_{8}(M W)$ & 22.15 & 22.63 & 22.05 & 21.8115 & 22.262 & 23.732 \\
\hline$P G_{11}(M W)$ & 12.14 & 12.928 & 12.44 & 12.3387 & 12.46 & 11.648 \\
\hline$P G_{13}(M W)$ & 12 & 12 & 12 & 12.0129 & 12 & 12 \\
\hline $\begin{array}{l}\text { Total } \\
\text { generation(MW) }\end{array}$ & 292.9 & 292.79 & 292.85 & 292.9105 & 292.886 & 292.734 \\
\hline Cost $(\$ / h)$ & 802.4 & 802.62 & 802.29 & 802.465 & 802.394 & 802.224 \\
\hline Losses (MW) & 9.48 & - & - & - & 9.466 & 9.391 \\
\hline
\end{tabular}

Table 4. Comparison of proposed algorithm with PSO, GA and SLFA algorithms (Cost)

\begin{tabular}{lcccccccc}
\hline Method & $P_{G 1}(M W)$ & $P_{G 2}(M W)$ & $P_{G 5}(M W)$ & $P_{G 4}(M W)$ & $P_{G 11}(M W)$ & $P_{G 3}(M W)$ & Cost $(\$ / \mathrm{h})$ & Emission $($ ton $/ \mathrm{h})$ \\
\hline CLPSO & 174.7477 & 49.4962 & 21.1095 & 23.7328 & 11.6481 & 12.0000 & 802.2240 & 0.3721 \\
SLFA & 179.0337 & 49.2580 & 20.3183 & 21.3269 & 11.5420 & 11.6655 & 802.5092 & 0.3720 \\
GA & 183.1419 & 46.2438 & 21.9576 & 17.2385 & 11.7363 & 13.0954 & 803.2332 & 0.3829 \\
PSO & 179.3912 & 48.4882 & 19.8833 & 20.7275 & 12.7280 & 12.0000 & 802.8351 & 0.3725 \\
\hline
\end{tabular}




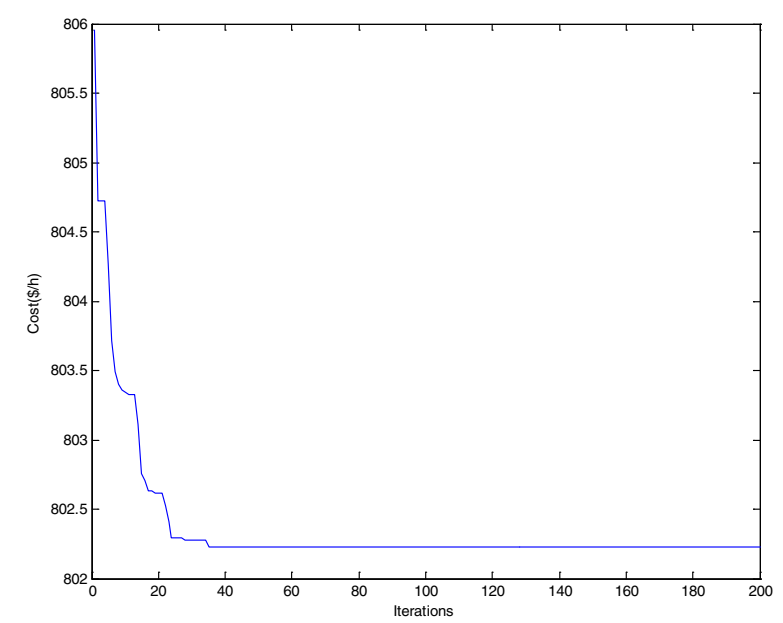

Figure 3. Convergence plot for CLPSOA for cost function.

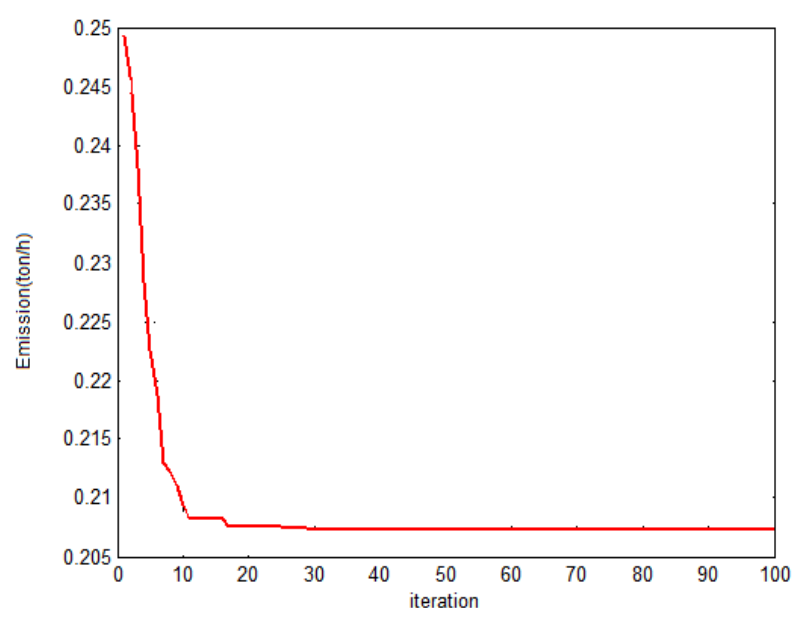

Figure 4. Convergence plot for CLPSO for emission function.

Table 5. Comparison of proposed algorithm with PSO, GA and original SLFA algorithms (Emission)

\begin{tabular}{llllllllc}
\hline Method & $P_{G 1}(M W)$ & $P_{G 2}(M W)$ & $P_{G 5}(M W)$ & $P_{G 8}(M W)$ & $P_{G 11}(M W)$ & $P_{G 13}(M W)$ & Cost $(\$ / \mathrm{h})$ & Emission $($ Ton $/ \mathrm{h})$ \\
\hline CLPSO & 65.3083 & 72.9975 & 50 & 34 & 28 & 40 & 957.6437 & 0.2059 \\
SLFA & 64.4840 & 71.3807 & 49.8573 & 35 & 30 & 39.9729 & 960.1911 & 0.2063 \\
GA & 78.2885 & 68.1602 & 46.7848 & 33.4909 & 30 & 36.3713 & 936.6152 & 0.2117 \\
PSO & 59.8079 & 80 & 50 & 35 & 27.1398 & 40 & 975.0797 & 0.2096 \\
\hline
\end{tabular}

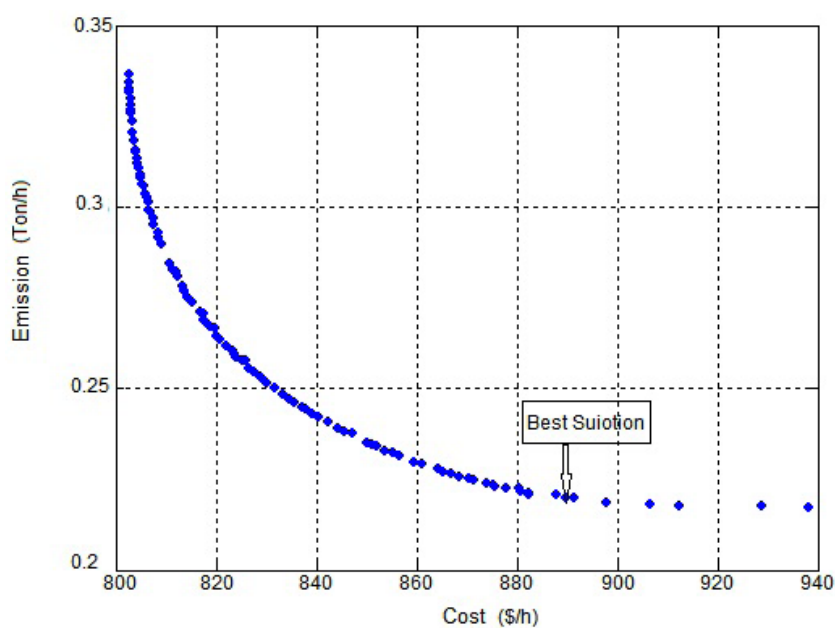

Figure 5. Some of the best non-dominated results.

\section{Conclusion}

The CLPSO based approach presented in this paper was applied to optimal power flow problem formulated as multi-objective optimization problem with competing objectives of fuel cost, emission. The results show the potential and efficiency of the proposed algorithm to solve multi-objective OPF problem and produce multiple Pareto-optimal solutions in one simulation run. In addition, the diversity and well-distribution characteristics of the non-dominated solutions obtained by the proposed algorithm have been demonstrated. The simulation results also reveal the superiority of the proposed algorithm in terms of the diversity and quality of the obtained Paretooptimal solutions. The approach was tested on the IEEE 30-bus 6-generator system. The results using the proposed approach were compared to those reported in the literature. The results confirm the potential of the proposed approach and show its effectiveness and superiority over the classical techniques and heuristic method.

\section{References}

1. Dommel HW, Tinney WF. Optimal power flow solutions, IEEE Transactions on Power Apparatus and Systems. 1968; PAS-87(10):1866-76.

2. Momoh JA, El-Hawary ME, Adapa R. A review of selected optimal power literature to 1993. Part I: non-linear and quadratic programming approaches. IEEE Transactions on Power Systems. 1999; 14(1):96-104. 
3. James AM, El-Hawary ME, Adapa R. A review of selected optimal power literature to 1993. Part II: Newton, linear programming and interior point methods. IEEE Transactions on Power Systems. 1999; 14(1):105-11.

4. Ambriz-Perez H, Acha E, Fuerte-Esquivel CR, De La Torre A. Incorporation of a UPFC model in an optimal power flow using Newton's method. IEEE Proceedings on Generation Transmission Distribution. 1998; 145(3):336-44.

5. Momoh JA, Guo SX, Ogbuobiri EC, Adapa R. The quadratic interior point method solving power system optimization problems. IEEE Transactions on Power Systems. 1994; 9(3):1327-36.

6. Lin QG, Huang GH. A dynamic inexact energy systems planning model for supporting greenhouse-gas emission management and sustainable renewable energy. Renew Sustain Energ Rev. 2009; 13(8):1836-53.

7. Roa-Sepulveda CA, Pavaz-Lazo BJ. A solution to the optimal power flow using simulated annealing. Int J Electr Power and Energ Sys. 2003; 25(1), 47-57.

8. El Elaa AAA, Abidob MA, Spea SR. Optimal power flow using differential evolution algorithm. Elec Power Syst Res. 2010; 80(7):878-85.

9. Osman MS, Abo-Sinna MA, Mousa AA. A solution to the optimal power flow using genetic algorithm. Appl Math Comput. 2004; 155(3):391-405.

10. Abido MA. Optimal power flow using particle swarm optimization. Int J Electr Power and Energ Syst. 2002; 24, 563-71.

11. Jeganathan C, Roy PS, Jha MN. Multi-objective spatial decision model for land use planning in a tourism district of India. Journal of Environmental Informatics. 2011; 17(1):15-24.

12. Birant D. Comparison of decision tree algorithms for predicting potential air pollutant emissions with data mining models. Journal of Environmental Informatics. 2011; 17(1):46-53.

13. Niknam T, Narimani MR, Jabbari M, Malekpour MR. A modified shuffle frog leaping algorithm for multi-objective optimal power flow. Energy. 2011; 36(11):6420-32.
14. Abido MA. Environmental/economic power dispatch using multiobjective evolutionary algorithms. IEEE Transactions Power System. 2003; 18(4):1529-37.

15. IEEE 30-bus test system data. Available from: www. ee.washington.edu/

16. Abido MA. A niched Pareto genetic algorithm for multiobjective environmental/ economic dispatch, .Electr. Power Energy Syst. 2003; 25(2):97-105.

17. Abido MA. Multiobjective evolutionary algorithms for electric power dispatch problem. IEEE Transactions Evolutionary Computations. 2006; 10(3):315-29.

18. Zhao B, Cao YJ. Multiple objective particle swarm optimization technique for economic load dispatch. J. Zhejiang Univ. SCIE. 2005; 6(5):420-27.

19. Liang JJ, Qin AK, Suganthan PN, Baskar S. Comprehensive learning particle swarm optimizer for global optimization of multimodal functions, IEEE Transactions on Evolutionary Computation. 2006; 10(3):281-295.

20. Mahadevan K, Kannan PS. Comprehensive learning particle swarm optimization for reactive power dispatch. Applied Soft Computing. 2010; 10(2):641-52.

21. Matpower Matlab toolbox. Available from: http://matpower.com/

22. Alsac O, Stott B. Optimal power flow with steady state security.IEEETransactionsonPower ApparatusandSystems, 1974; 93(3):745-51.

23. Yuryevich J, Wong KP. Evolutionary programming based optimal power flow algorithm. IEEE Transactions on Power Systems. 1999; 14(4):1245-50.

24. Abido MA. Optimal power flow using Tabu search algorithm. Elec Power Compon Syst. 2002; 30(5):469-83.

25. Ongsakul W, Tantimaporn T. Optimal power flow by improved evolutionary programming. Elec Power Compon Syst. 2006; 34(1):79-95.

26. Sayah S, Zehar K. Modified differential evolution algorithm for optimal power flow with non-smooth cost functions. Energ Convers Manag. 2008; 49(11):3036-42. 